Claudio M. Pacella, MD

Giancarlo Bizzarri, MD

Fabrizio Magnolfi, MD

Piero Cecconi, MD

Bruno Caspani, MD

Vincenzo Anelli, MD

Antonio Bianchini, MD

Dario Valle, MD

Sara Pacella, MD

Guglielmo Manenti, MD

Zaccaria Rossi, MD

Index terms:

Lasers, interstitial therapy, 761.1299

Liver, interventional procedures,

761.1299

Liver neoplasms, 761.323

Liver neoplasms, therapy, 761.1266

$761.1299,761.323$

Published online before print

$10.1148 /$ radiol.2213001501

Radiology 2001; 221:712-720

Abbreviations:

HCC = hepatocellular carcinoma

$\mathrm{LTA}=$ laser thermal ablation

$\mathrm{PEI}=$ percutaneous ethanol injection

${ }^{1}$ From the Departments of Radiology and Diagnostic Imaging (C.M.P., G.B., V.A., A.B., D.V., S.P., G.M.) and Endocrine, Metabolic, and Digestive Diseases (Z.R.), Regina Apostolorum Hospital, Via St Francesco 50, 00041 Albano Laziale, Rome, Italy; the Gastroenterology Unit, St Donato Hospital, Arezzo, Italy (F.M.); and the Diagnostic Radiology Service, St Anna Hospital, Como, Italy (P.C., B.C.).

From the 2000 RSNA scientific assembly. Received September 6, 2000; revision requested October 20; final revision received May 7, 2001; accepted June 5. Address correspondence to C.M.P. (e-mail: cmpacel@katamail.com).

○ RSNA, 2001

Author contributions:

Guarantor of integrity of entire study, C.M.P.; study concepts, C.M.P., G.B., Z.R.; study design, C.M.P., G.B., P.C.; literature research, S.P., G.M.; clinical studies, F.M., Z.R.; data acquisition, A.B., S.P.; data analysis/interpretation G.B., F.M., P.C., B.C., A.B.; statistical analysis, D.V., S.P., G.M., C.M.P., A.B.; manuscript preparation, C.M.P., S.P.; manuscript definition of intellectual content, all authors; manuscript editing, A.B., S.P.; manuscript revision/review, C.M.P., P.C., B.C., Z.R.; manuscript final version approval, all authors.

\section{Laser Thermal Ablation in the Treatment of Small Hepatocellular Carcinoma: Results in 74 Patients ${ }^{1}$}

PURPOSE: To evaluate the safety, local effectiveness, and long-term results of laser thermal ablation (LTA) in the treatment of small hepatocellular carcinoma (HCC).

MATERIALS AND METHODS: Ninety-two biopsies proved small HCCs (range, $0.8-4.0 \mathrm{~cm}$ ) in 74 patients who were treated percutaneously with LTA in an outpatient clinic. A laser at a power of $5.0 \mathrm{~W}$ was coupled with one to four fibers that were advanced through 21-gauge needle(s) for 6-12 minutes. All lesions were evaluated with computed tomography (CT) for changes in size and vascular pattern, recurrence rates, and cumulative survival rates. Patients were examined for complications.

RESULTS: No major complications occurred in 117 LTA sessions, with an average of 1.3 sessions per tumor. At 3 months, CT scans showed a nonenhancing area (complete necrosis) in 89 (97\%) of 92 lesions. During follow-up (range, 6-66 months; mean, 25.3 months), 84 tumors (91\%) decreased in size. The local recurrence rates (range, $1-5$ years) ranged from $1.6 \%$ to $6.0 \%$. Recurrence rates (range, 12-60 months) in other liver segments ranged from $24 \%$ to $73 \%$. Cancer-free survival rates (range, $1-4$ years) ranged from $73 \%$ to $24 \%$. Overall survival rates were $99 \%, 68 \%$, and $15 \%$ at 1,3 , and 5 years, respectively. Twenty-one patients (28\%) died.

CONCLUSION: LTA is a safe and effective treatment for small HCC.

Early detection strategies for hepatocellular carcinoma (HCC) have increased the number of small HCCs that are treatable with resection in countries where this disease is endemic $(1,2)$. The survival rates reported $(3,4)$ after partial resection or subsegmentectomy have improved in the past decade, mainly because of the ever smaller tumors undergoing resection. However, resection is possible in only a small proportion of patients because of the underlying advanced cirrhosis (4). Furthermore, it has been observed that HCC frequently recurs in the liver remnant after resection (5). Orthotopic liver transplantation is also an effective treatment for small unresectable HCCs when donors are available for potential recipients (6).

These problems in the management of HCC prompted the development of other potentially therapeutic modalities, such as transcatheter arterial embolization (7); transcatheter arterial chemoembolization (8); percutaneous cryotherapy (9); and ultrasonographically (US) guided percutaneous injection of hot saline (10), acetic acid (11), or ethanol (12), all of which destroy cancer cells. Transcatheter arterial chemoembolization does not eliminate all malignant cells, and although extensive tumor necrosis can be observed, viable residual tumor cells remain, particularly beneath the tumor capsule, in intracapsular invasion, in daughter nodules, and in portal venous tumor thrombi $(8,13)$. Percutaneous ethanol injection (PEI) is now widely used for small HCC $(12,14)$. The effect of PEI is roughly comparable to that of surgical resection $(5,12)$. However, a larger number of treatment sessions is required (12), and PEI does not always produce complete necrosis $(13,14)$. Furthermore, recurrence after treatment is very common $(12,15-17)$, and needle track implantation is not unusual, especially when HCCs are $2 \mathrm{~cm}$ or greater at their largest diameter (18); in addition, sclerosing HCCs respond poorly to PEI therapy (19). 


\begin{tabular}{|c|c|}
\hline \multicolumn{2}{|l|}{ Baseline Characteristics of Patients } \\
\hline Patient Characteristic & Value \\
\hline No. of patients & 74 \\
\hline No. of lesions & 92 \\
\hline Age range $(y)$ & $52-80$ \\
\hline Mean age $(y) \pm S D$ & $67.5 \pm 7.7$ \\
\hline $\operatorname{Sex}(M: F)$ & $47: 27$ \\
\hline Origin of disease (VHC, VHB, VHC + VHB, cryptogenetic, or alcohol-related) & $48: 14: 3: 2: 7$ \\
\hline Mean serum bilirubin $(\mu \mathrm{mol} / \mathrm{L}) \pm S D$ & $18.8 \pm 10.3$ \\
\hline Mean serum albumin $(\mathrm{g} / \mathrm{L}) \pm \mathrm{SD}$ & $32 \pm 5$ \\
\hline Mean prothrombin activity (INR) $\pm S D$ & $1.17 \pm 5.74$ \\
\hline Child-Pugh score A or B & $54: 20$ \\
\hline Tumor stage solitary or multinodular & $58: 16$ \\
\hline Ratio of tumor of $0.8-1.0,1.1-2.0,2.1-3.0,3.1-4.0 \mathrm{~cm}$ & $5: 24: 51: 12$ \\
\hline \multicolumn{2}{|l|}{ Diameter of tumor $(\mathrm{cm})$} \\
\hline Range & $0.8-4.0$ \\
\hline Mean \pm SD & $2.4 \pm 0.7$ \\
\hline \multicolumn{2}{|l|}{ Volume of tumor $(\mathrm{mL})$} \\
\hline Range & $0.5-30$ \\
\hline Mean \pm SD & $8.2 \pm 6.1$ \\
\hline Tumor stain present, absent, or mixed & 78:7:7 \\
\hline Tumor location unilobar or bilobar & $87: 5$ \\
\hline Tumor segment location $2,3,4,5,6,7$, or 8 & $4: 7: 13: 9: 30: 9: 20$ \\
\hline $\begin{array}{l}\text { US pattern hypoechoic, hyperechoic, isoechoic with hypoechoic rim, or } \\
\text { mixed }\end{array}$ & $74: 5: 7: 6$ \\
\hline Tumor capsule present, absent, or irregular & $38: 46: 8$ \\
\hline $\begin{array}{l}\text { Histologic grade of tumor differentiation well differentiated, moderately } \\
\text { differentiated, poorly differentiated, undifferentiated, or mixed }\end{array}$ & $43: 39: 8: 1: 1$ \\
\hline No. of patients with serum AFP value $<20,20-200$, or $>200(\mu \mathrm{g} / \mathrm{L})$ & 54:15:5 \\
\hline
\end{tabular}

To improve the results obtained with PEI, other percutaneous techniques have been developed. The most promising minimally invasive techniques are hyperthermic methods of tissue destruction, such as radio-frequency electrocoagulation (20-22), microwave coagulation (23), and laser thermal ablation (LTA) (24-29). Each of these techniques generates intralesional heat by a different mechanism, but the local hyperthermia is the unifying mechanism underlying their effectiveness. To date, all of these new percutaneous lesion-heating techniques are less well established than PEI.

The purpose of our work was (a) to evaluate the safety of LTA in the treatment of small HCCs, (b) to investigate the local effectiveness of this technique, and (c) to analyze the long-term results in a series of 76 patients.

\section{MATERIALS AND METHODS}

Between March 1994 and December 1999, we enrolled 74 patients (92 lesions) with a single tumor $4 \mathrm{~cm}$ or smaller or with one to three nodules $3 \mathrm{~cm}$ or smaller, among 308 patients with HCC diagnosed and staged at the Regina Apostolorum hospital. All patients were poor surgical candidates or had unresectable lesions. Exclusion criteria were age older than 80 years, evidence of extrahepatic metastases (based on the coincident findings of at least two imaging techniques) and/or lobar and local portal venous thrombosis, uncontrolled liver disease decompensation (gastrointestinal bleeding, encephalopathy, refractory ascites, bacterial infection), clotting impairment (platelet count less than $40 \times 10^{9} / \mathrm{L}$ or prothrombin time with international normalized ratio, or INR, greater than 1.99 seconds) (22), renal failure, or ChildPugh class C cirrhosis. Patients who fulfilled the criteria gave their written informed consent to enter the study, which had been approved by the investigation and ethics committee of the hospital. The patients' baseline characteristics are summarized in the Table.

Histologic grading of the neoplasm was confirmed in all cases at US-guided percutaneous biopsy with a continuous real-time scanner (AU 590 Asynchronous, AU 4 Idea; Esaote Biomedica, Genoa, Italy) by using an 18-gauge cutting needle (Biomol; Hospital Service, Rome, Italy). In all patients, we performed an $\alpha$-fetoprotein assay. The size, number and location of lesions in the liver, vascular and biliary ingrowth, and regional lymphatic involvement were es- tablished at US and computed tomography (CT). CT was performed with a helical scanner (HiSpeed Advantage; GE Medical Systems, Milwaukee, Wis). In all patients, triple-phase scans were obtained starting 25, 70, and 180 seconds after the powered injection (CT 9000; Liebel-Flarsheim, Cincinnati, Ohio) of a single bolus of a solution of $150 \mathrm{~mL}$ of iopromide (Ultravist 370; Schering, Berlin, Germany) in water at a rate of $5 \mathrm{~mL} /$ $\sec (29)$.

The presence of a tumor capsule (complete hyperattenuating ring surrounding the lesion[s] during equilibrium phase) was established on pretreatment contrast material-enhanced CT scans. The lesions that were surrounded by a capsule or with smooth, well-circumscribed margins without a capsule (absence of hyperattenuating ring surrounding the lesion[s] during equilibrium phase) were considered noninfiltrating. The lesions with irregular margins or encapsulated lesions with extranodular growth (irregular capsule) and/or peripheral satellite nodules were classified as infiltrating.

On the basis of the behavior of the lesions on pretreatment contrast-enhanced CT scans obtained during the arterial dominant phase, the tumors were classified as hyperattenuating (presence of tumor stain), hypoattenuating (absence of tumor stain), or a mixed pattern (inhomogeneous pattern). The US texture of the lesion(s), as compared with that of the surrounding liver parenchyma, was classified as hypoechoic, hyperechoic, isoechoic with hypoechoic rim, or mixed. The volume (the three largest perpendicular diameters of the lesion multiplied by 0.525 [ellipsoid volume]) of each tumor was assessed from the CT image by one radiologist (C.M.P.). Seven nodules were superficially located and/or were beneath the capsule; three, close to the gallbladder; two, near the porta hepatis; ten, near the hepatic veins; two, near the mediumsized vessels; and none, adjacent to the vena cava. Two nodules in the third segment deformed the liver margin (Table).

\section{LTA Technique}

Preparation for LTA was performed with the same guidelines as those used for large-core percutaneous biopsy. All patients were treated in our outpatient clinic. Percutaneous treatment is performed in the angiography suite by one radiologist in the simpler cases and by two radiologists in more complex and harder-to-reach cases, with one nurse who is experienced in dealing with con- 
scious sedation during interventional procedures (30). Local anesthesia was achieved by using $5 \mathrm{~mL}$ of $2 \%$ xilocaine (Xilocaine $2 \%$; AstraZeneca, Basiglio, Italy). Just before needle insertion, both 3-7 $\mathrm{mg}$ of midazolam maleate (Ipnovel 15; Roche, Milan, Italy) and $4 \mathrm{~mL}$ of fentanyl citrate (Fentanest; Pharmacia \& Upjohn, Milan, Italy) were administered intravenously while the cardiovascular and respiratory systems were continuously monitored. With US guidance and a $3.5-\mathrm{MHz}$ convex transducer (CAB411A-EZU-PA3B4-1; Esaote Biomedica), the needles were positioned within the lesion in the area to be treated, and the tip positions were confirmed with two-plane US images.

LTA was performed with a continuouswave neodymium yttrium-aluminiumgarnet, or Nd:YAG, laser (DEKA-M.E.L.A.; Florence, Italy) that operated at a wavelength of $1.064 \mu \mathrm{m}$. This laser source was used with an optical beam-splitting device (SMART 1064 HCC; DEKA-M.E.L.A.) with four separate fibers that were always illuminated concurrently (simultaneous or multiple-fiber application) (31). A 21gauge modified Chiba needle (Hospital Service) and a plane-cut tip fiberoptic with a $300-\mu \mathrm{m}$ quartz core (Medical Energy, Pensacola, Fla) were used. The needles were placed within the tumor prior to starting treatment, and each stylet was replaced by a sterile optical fiber, which was advanced until at least $1 \mathrm{~cm}$ of the bare fiber tip was in direct contact with the deepest part of the tumor. Both the number of needles (one to four) and the arrangement were chosen in accordance with the size, shape, and location of the lesions.

To achieve regional coagulation with necrosis of the main tumor, capsular invasion, and neighboring intrahepatic metastases (peripheral satellite nodules and portal vein thrombi), up to four needles were simultaneously placed in a square configuration with interneedle spacing of $1.0-1.5 \mathrm{~cm}$, encompassing the whole tumor and surrounding noncancerous parenchyma, with the intention of creating a volume of necrosis larger (a rim at least $5-10 \mathrm{~mm}$ of the rim of normal liver around the tumor) than the original volume of the neoplasm. Once the laser was turned on and the scheduled energy was delivered for the single treatment, if warranted by the size of the lesion, another laser treatment was performed by withdrawing the needles and fiber tips $2.0 \mathrm{~cm}$ from the treated area and carefully repositioning them in the contiguous, still untreated cranial area within the lesion. All laser illuminations were monitored continuously with realtime US. At the end of the treatment, the optic fiber was slowly pulled out with the laser still on. Once the needles were pulled out, US was performed to reveal intraand/or extrahepatic complications. LTA sessions were performed by one or two of five radiologists (C.M.P., G.B., F.M., P.C., B.C.) with at least 5 years experience in interventional abdominal procedures and fellowship training in our hospital.

After the procedure, patients were observed for 6 hours in the outpatient recovery room and, if no complications occurred, were discharged. Before the procedure and at 6 and 24-48 hours and 1 month after treatment, the following serologic values were measured: levels of transaminases, alkaline phosphatase, bilirubin, electrolytes, hemoglobin, fibrinogen, haptoglobin, and creatinine; prothrombin activity; and complete blood cell count. The degree of liver function impairment was estimated by means of routine biochemical parameters within 2 months after LTA. In addition, recorded data included Child-Pugh classification, growth of tumor, and occurrence of extrahepatic disease.

\section{Study Design and Assessment of Treatment Effectiveness}

Our aim was to achieve a rapid, safe, and complete destruction of small HCCs by producing a large area of necrosis while markedly reducing the number of sessions required to carry out a complete ablation. On the basis of our experience (32) and that reported by other authors (33), we used $5 \mathrm{~W}$ per fiber with an exposure time of 6 minutes to reach $1,800 \mathrm{~J}$ per fiber for each single tumor treatment. A laser illumination that lasted 6 minutes was considered a single tumor treatment. Up to two treatments were scheduled during any one session. We scheduled no more than two irradiation cycles for any one lesion, depending on the size of the tumor. The first irradiation cycle was composed of a maximum of two sessions and a maximum of two tumor treatments per session; and the second irradiation cycle, with the same design as the first cycle, took place after 3 months if there was incomplete necrosis.

We used one fiber for lesions less than or equal to $1 \mathrm{~cm}(n=5)$, two fibers for lesions less than or equal to $2 \mathrm{~cm}$ ( $n=$ 24), and four fibers for lesions with a diameter of $2.1-4.0 \mathrm{~cm}(n=63)$. Thus, we determined the number of LTA tumor treatments and sessions, the total energy used per session and per tumor, the mean volume of necrosis obtained and the energy delivered per milliliter of tumor, and the mean total procedure time.

During and at 5-6 hours and 24-48 hours after treatment, US was performed to evaluate any changes in the echotexture of the lesions treated and in thermally induced necrosis. After 1-2 weeks and every 6 months thereafter, US was performed to monitor changes in size and lesion detectability during follow-up.

CT was performed 24-48 hours after the first irradiation cycle to evaluate the short-term effects of the procedure, assess the extent of necrosis, and plan further intervention. Still-enhancing areas were assumed to indicate residual viable tumor, and an additional irradiation cycle (ie, one or two sessions of LTA) was performed. Thus, tumor necrosis was considered complete when no areas of enhancement were seen in the tumor or at the periphery on CT scans obtained 3 months after the first irradiation cycle or 24-48 hours after a possible second irradiation cycle. If CT scans obtained after the second irradiation cycle showed residual viable tissue, LTA was considered to have failed and was discontinued; in our hospital, patients then underwent segmental transcatheter arterial chemoembolization within 30-60 days after LTA failure.

On the basis of the findings of this imaging technique, the response to LTA was defined in accordance with World Health Organization criteria as complete response, no evidence of neoplastic disease; partial response, reduction greater than 50\%; no change, reduction less than $50 \%$; and progressive disease, increase of greater than 25\% (34). Local recurrence was diagnosed when enhancing foci were seen close to, along the edge of, or within the treated area. CT was performed 3 months after the end of treatment and every 6 months thereafter. All CT scans were analyzed in consensus by two experienced radiologists (C.M.P., D.V.). Therapeutic effectiveness was also assessed with an $\alpha$-fetoprotein assay performed after the end of LTA and every 6 months thereafter. During follow-up, hepatic serum functional indexes were measured every 6 months.

\section{Statistical Analyses}

Statistical analysis was conducted every 6 months. The patients' baseline characteristics and follow-up results are presented as means plus or minus SDs. Rates of local recurrence, occurrence of new lesions, death, cancer-free survival, and cu- 

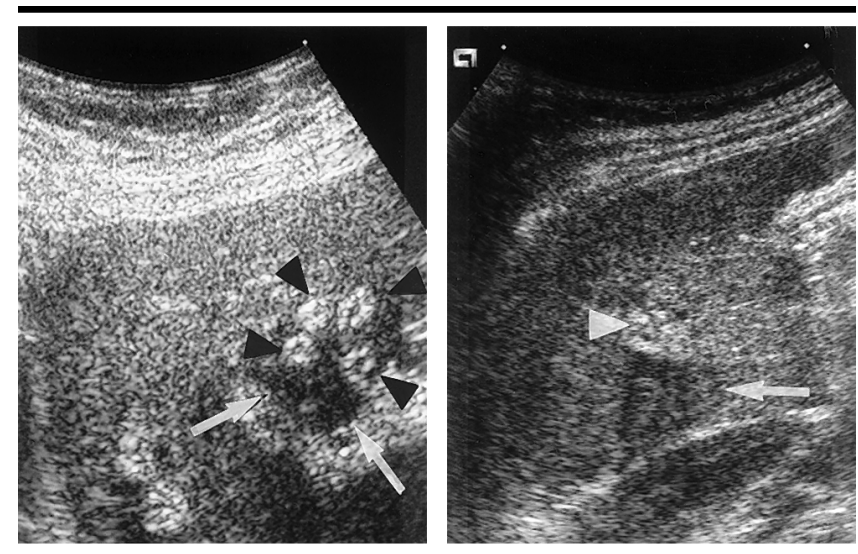

a.

b.

Figure 1. (a) Transverse US image obtained 24 hours after LTA shows four thermal lesions characterized by a small central hypoechoic area (zone of vaporization) that is surrounded by a hyperechoic rim (zone of carbonization) (arrowheads) and an outer broad hypoechoic area (zone of coagulative necrosis) (arrows). (b) Longitudinal US image obtained 24 months after LTA shows an ill-defined hyperechoic area (arrowhead) with associated acoustic shadowing (arrow).

mulative survival were calculated from the time of initial treatment of the original HCC by using the Kaplan-Meier method. Comparison was made by performing the log-rank test. $P$ values of less than .05 were considered to indicate a statistically significant difference.

\section{RESULTS}

A total of 196 tumor treatments (range, one to six treatments; median, two; mean, 2.1) were performed during 117 sessions (range, one to three sessions; median, one; mean, 1.3). The total energy used at each session was 1,800-15,900 J (median, $8,000 \mathrm{~J})$. The energy used per tumor was 3,600-44,600 J (median, 12,500 J). The mean volume of necrosis obtained was $15.2 \mathrm{~mL} \pm 9.5(\mathrm{SD})$ (range, 2-44 mL), with a total energy deposition of 800 $\mathrm{J} / \mathrm{mL}$ of tumor (range, $350-1,600 \mathrm{~J} / \mathrm{mL}$; median, $700 \mathrm{~J} / \mathrm{mL}$ ). The mean total procedure time was 25.5 minutes \pm 7.9 per session (range, 15-45 minutes).

\section{Imaging}

Laser illuminations were monitored continuously with real-time US. In every case, once LTA treatment had begun, continuous US monitoring revealed, after a delay of about 30-60 seconds, a slowly enlarging and coalescing hyperechoic zone around each fiber tip resulting from vaporization of fluid and formation of microbubbles of gas. This irregular and poorly defined hyperechoic area then progressively increased to envelop the entire tumor. Vapor appeared as echogenic linear foci radiating from the treatment area along tissue planes and/or hepatic vessels or coming up through the needle sheath and becoming visible as smoke in the needle hub. US performed after treatment depicted a decrease in the echogenicity of the treated area, and after 5-6 hours, a heterogeneous lesion was surrounded by a 3-5-mm hypoechoic rim. At US performed after 24-48 hours, the thermal lesion was characterized by a small central hypoechoic area (zone of vaporization), which was surrounded by a thin hyperechoic rim (zone of carbonization) and a thick outer hypoechoic area (zone of coagulation) with associated acoustic shadowing (32) (Fig 1). This US appearance was nearly identical to that on 1-2-week and 6- and 12-month follow-up images, apart from a decrease in size of both the central cavity and the entire heated area. These changes precluded precise assessment of the coagulation size after LTA.

On precontrast CT scans, the entire treated tumor was replaced by an irregular area of hyperattenuation that was surrounded by a thin rim of lower attenuation (the "ghost" of the original neoplasm) (Fig 2). On contrast-enhanced CT scans obtained after treatment, the whole heated region appeared as a uniform area of low attenuation that was surrounded by a rim of higher attenuation (Fig 2), which disappeared gradually during follow-up. In five
(5\%) of 92 cases, the area of hyperattenuation that surrounded the coagulative necrosis had, after 24 hours, a segmental configuration (Fig 3).

All 84 (91\%) successfully treated tumors had diminished in size by $65 \%$ and $87 \%$ at 6 and 12 months, respectively, after LTA. The nonenhancing areas at the site of the treated HCC remained detectable as small hyperechoic areas with acoustic shadowing at US follow-up examination (Fig 1) and as small areas of hypoattenuation during the arterial (Fig 2) and equilibrium phases on follow-up CT scans obtained 24-36 months after treatment. The coagulation areas were no longer detectable at follow-up CT and on US images obtained 36-48 months after treatment. In our series, two lesions were still detectable after 48 and 60 months and had the characteristics just described.

\section{Therapeutic Responses and Follow-up Findings}

A single irradiation cycle was performed in $84(91 \%)$ of 92 lesions. In eight (9\%) tumors, a second irradiation cycle that consisted of only one session was performed (Fig 4). We obtained a volume of necrosis larger than that of the original lesion in 77 (84\%) of 92 tumors; in 12 (13\%) tumors, we achieved a volume equal to the initial volume and corresponding to the original lesion in size and shape (Fig 4); in the remaining three $(3 \%)$ tumors, we observed evidence of tumor persistence (partial response). Complete response (complete necrosis) was achieved in 89 (97\%) of 92 tumors. Of the three tumors (two patients) in which necrosis was incomplete, one was of grade 2 and located in the fifth segment and two were of grade 3 and located in the eighth segment. In both tumors, transcatheter arterial chemoembolization was performed; in one after 32 and in the other 55 days after the last LTA treatment.

During follow-up (range, 6-66 months; mean, 25.3 months), five (6\%) of 89 successfully treated lesions showed a local recurrence. The 1-, 2-, and 5-year local recurrence rates were $1.6 \%, 6.0 \%$, and $6.0 \%$, respectively, and all local recurrences were observed within 2 years after LTA (range, 8-22 months; mean, 14.8 months) (Fig 5). A new HCC (one or more lesions with diameter of $0.8-3.0 \mathrm{~cm}$; mean, $2.1 \mathrm{~cm}$ ) occurred elsewhere in the liver in 36 (49\%) of 74 patients within 3-64 months (mean, 17.7 months) after LTA. The 12-, 24-, 36-, 48-, and 60-month recurrence rates in segments other than that of the original site were $24 \%, 45 \%, 62 \%, 73 \%$, and $73 \%$, re- 
spectively. These recurrences were usually treated with additional courses of LTA in patients $(n=20)$ with one to three new lesions or with transcatheter arterial chemoembolization in patients $(n=5)$ with more than three new lesions. The remaining patients $(n=11)$ did not undergo further treatment because they developed either severe liver decompensation $(n=6)$, disseminated neoplastic disease $(n=3)$, or were in poor general condition $(n=2)$. Cancer-free survival rates, including both local recurrences and new lesions, were $73 \%, 50 \%, 36 \%$ and $24 \%$ at $1,2,3$, and 4 years, respectively.

Cumulative survival rates were 99\%, $95 \%, 68 \%, 45 \%$, and $15 \%$ at $1,2,3,4$, and 5 years, respectively (Fig 6). In patients with Child-Pugh class A, the survival rates were $100 \%, 97 \%, 73 \%, 62 \%$, and $31 \%$ at $1,2,3,4$, and 5 years, respectively. These figures show no statistically significant difference $(P=.052)$ from those obtained in patients with ChildPugh class B, who had survival rates of $95 \%, 89 \%, 68 \%, 29 \%$, and $0 \%$ at 1, 2, 3, 4 , and 5 years, respectively (Fig 7). Serum $\alpha$-fetoprotein levels returned to normal after LTA in all patients who had had a slightly increased serum $\alpha$-fetoprotein level and in four (80\%) of five patients who had had a serum level over 200 $\mathrm{ng} / \mathrm{mL}(200 \mu \mathrm{g} / \mathrm{L})$ before therapy. In the remaining patient, the serum $\alpha$-fetoprotein level remained high due to a new HCC elsewhere in the liver.

During follow-up, 21 (28\%) of 74 patients died. The cause of death was HCC in nine (43\%) patients, hepatic failure in nine $(43 \%)$, cardiovascular disease in two (10\%), and primary lung neoplasm in one (5\%). Lung metastasis occurred in one (11\%) of nine cases of death due to HCC.

\section{Side Effects and Complications}

No major complications requiring intensive care were encountered during or after the procedure. Three minor complications in the course of 120 LTA sessions occurred in three patients during the first 6-24 hours after the procedure: self-remitting variceal bleeding (reduction of hemoglobin level by $8 \mathrm{~g} / \mathrm{dL}$ [80 g/L]), subcapsular hemorrhage, and perirenal inflammation. The first two complications required no treatment, although discharge from the outpatient recovery room was delayed, and hospital admission was indicated. Neither transfusion nor surgical repair was necessary for either the patient with variceal bleeding, since the hematocrit level returned to baseline values within 5 days, or the pa-
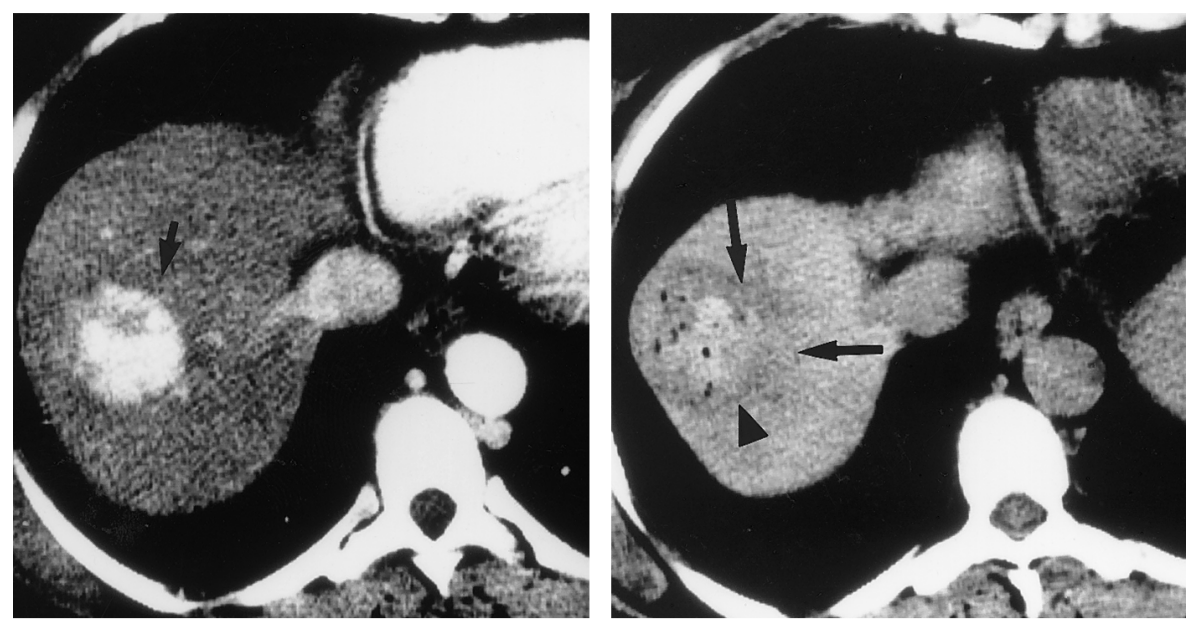

a.

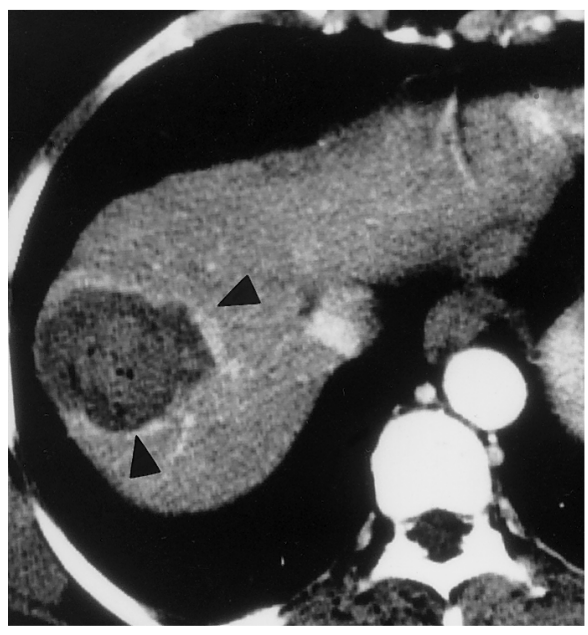

b.

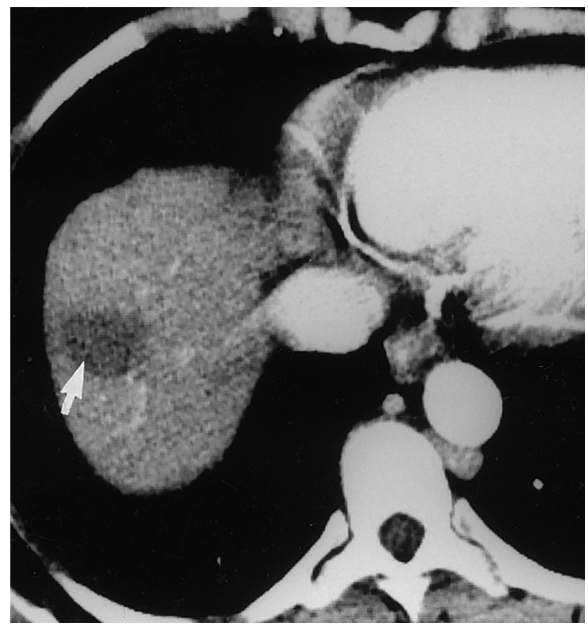

d.

Figure 2. CT scans show complete response after LTA (energy delivered, 28,800 J). (a) Transverse arterial phase CT scan obtained before LTA shows a hyperattenuating 3.7-cm-diameter nodular lesion (arrow) in segment 8. (b) Transverse precontrast CT scan obtained 24 hours after LTA shows that the entire tumor has been replaced by an irregular and ill-defined area of hyperattenuation (the "ghost" of the original neoplasm) (arrowhead) surrounded by a rim of lower attenuation (arrows). The small zones of vacuolation resulted from vaporization of fluid and formation of microbubbles of gas. (c) Transverse arterial phase contrast-enhanced CT scan obtained 24 hours after LTA shows the entire heated region characterized by a homogeneous area of hypoattenuation, due to coagulation, surrounded by a thin rim of hyperattenuation (arrowheads) due to hyperemia. The area of necrosis is larger than the original nodular lesion. (d) Transverse arterial phase CT scan obtained 24 months after LTA shows a marked reduction in size of the area of necrosis (arrow).

tient with intraparenchymal bleeding. In the third patient in whom the lesion was located peripherally, adjacent to the Glisson capsule in the sixth segment, we observed perirenal inflammation (hypoechoic irregular area at US and ill-defined area of hyperattenuation at contrast-enhanced CT), which cleared up in 20 days; the perirenal disease required the administration of $1 \mathrm{~g}$ of amoxicilline (Zimox; Pharmacia \& Upjohn) once a day for 7 days but not hospitalization.

All patients experienced mild to moderate pain at the insertion site or the epigas- tric level, or diaphragmal irritation with right shoulder tip pain during the procedure, especially when the tumor was superficial or contiguous to the porta hepatis. Sixty-one (82\%) of 74 patients experienced moderate pain or slight discomfort in the right hypochondrium during the next few days. In only $36(59 \%)$ of these cases did the pain require the administration of 500 mg of acetaminophen (Tachipirina 500; Angelini, Rome, Italy) twice a day; in five (8\%) cases, 10 drops of ketorolac tromethamine (Toradol; Recordati, Rome, Italy) was required three times a day in the fol- 


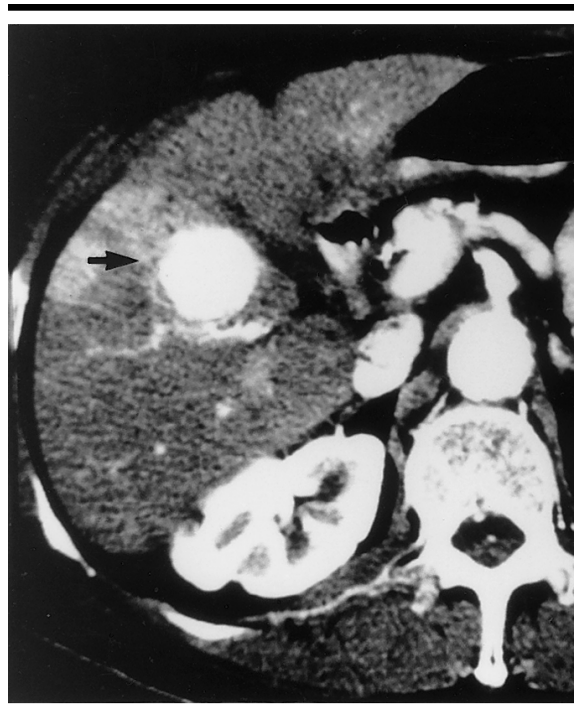

a.

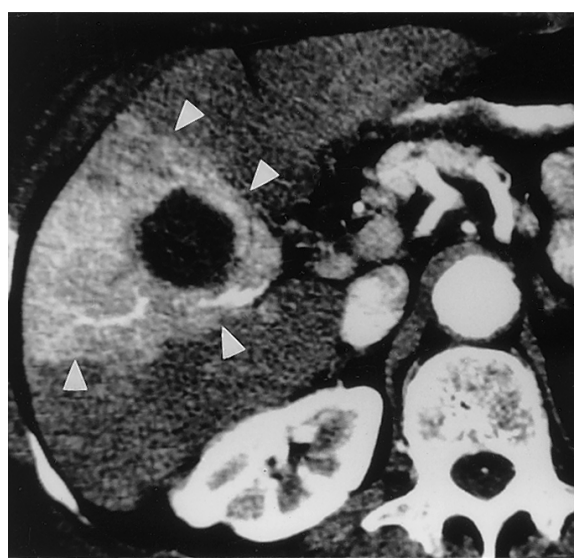

b.

Figure 3. CT scans show complete response after LTA (energy delivered, 28,800 J). (a) Transverse arterial phase CT scan obtained before LTA shows a hyperattenuating 3.5 -cm-diameter nodular lesion (arrow) in segment 4 . (b) Transverse arterial phase contrast-enhanced CT scan obtained 24 hours after LTA shows a homogeneous area of hypoattenuation surrounded by a segmental area of hyperattenuation (arrowheads).

lowing 3-4 days. Seventy (95\%) of 74 patients had a mild or moderate increase in body temperature $\left(37^{\circ}-38^{\circ} \mathrm{C}\right)$ without a focus infection, which returned to normal within 3-4 days. In 19 (50\%) of 38 patients in whom lesions were located in the dome of the liver, pleural effusion was observed on CT scans after 24 hours. None of these minor complications required treatment, and all cleared up after a few weeks. In all patients, transaminase levels were two to 10 times higher after treatment than at baseline, but they decreased to normal baseline values within 3-20 days. No changes in the serum level of total bilirubin and unconjugated bilirubin, complete blood cell count, or platelet count were observed. At the time this article was written, no evidence of needle-route seeding had been seen.

\section{DISCUSSION}

To our knowledge, our patient population was the first series of patients with small HCC to be treated percutaneously with LTA. Our study findings demonstrate that treatment with LTA can result in complete tumor necrosis in a high percentage of tumors. We hypothesized that in the three nodules with incomplete response, treatment failure could be explained by improper placement of the needles in the lesion. When simultaneous multiple fiber application is used, the distance at which the fibers are positioned determines whether the synergistic thermal effect between the fibers will occur $(31,35)$. Even a small deviation from the linearity of the insertion tract can cause either diverging fibers with insufficient tissue coagulation or converging channels with an accumulation of light and heat, causing subsequent tissue carbonization and fiber breakdown. If the optical fibers are correctly placed within the tumor, a thermal lesion that is larger than that obtained with PEI $(5,12,17,22)$ or with other heating percutaneous techniques $(22,23)$ invariably occurs.

An advantage of LTA is that it requires fewer treatment sessions for a complete treatment than does PEI $(12,22)$. Our results are comparable to those achieved with radio-frequency electrocoagulation (22), but with the latter method, the investigators excluded patients who had lesions adjacent to the hepatic hilum or confluence of the hepatic veins (22). The use of thin fibers and fine needles with LTA makes it possible to reach the entire lesion safely, even if it is very deep, difficult to get at, near the gallbladder or the hepatic hilum, or superficial. Moreover, LTA treatment seems to be extremely well tolerated and produces no severe side effects or major complications.

Our study findings show that local recurrence (Figs 5,8) does occur in small HCCs treated with LTA, but this drawback is infrequent. Other authors $(5,11,14-$ 16), by using PEI, report variable values that range from $4 \%$ to $30 \%$. In our opinion, this difference can be explained, at least in part, by the more complete necrosis that is achieved with LTA in most cases.

It has been shown that PEI therapy cannot be effective for intracapsular and extracapsular nodules. In fact, viable tumor tissue remains in portions isolated by septa, along the edge of the lesion, and in small nodules around the main lesion (14). PEI can eliminate the main tumor, but it cannot destroy any neighboring neoplastic or preneoplastic nests (36). Thus, HCC may recur after PEI treatment, not only because of incomplete necrosis of the main nodule but also because of the progressive growth of the neighboring nodules (36). Furthermore, the large number of treatment sessions required for PEI $(5,15-17,22)$ may increase the risk of tumor cell spread. In contrast, with one to two LTA treatment sessions, one can obtain a volume of necrosis larger than the original neoplasm with an adequate margin of security (surgical margin) around the lesion. Moreover, few authors (37) who reassessed preoperative diagnoses by examining resected liver specimens have observed that even the latest imaging techniques are not sensitive enough to depict all small HCCs. Thus, in our study, even the latest imaging techniques did not depict all of the small intratumoral nodules, intracapsular invasions, and extracapsular nodules, which will still be viable.

Cancer cells of this sort, which eluded percutaneous therapy, are perhaps the main cause of local recurrence. Most unrecognized intratumoral nodules, that is, intracapsular and extracapsular cancer cells that are undetectable at imaging and are not identified at pretreatment staging, may have a chance of being destroyed with LTA. In this way, in our opinion, LTA may be equivalent to limited hepatic resection and may influence long-term survival, achieving results comparable to those of segmentectomies. Confirmation can come only from controlled prospective trials in which both treatments are compared and the patients are followed up for at least 5 years.

It is difficult to compare reliably radiofrequency ablation with other heating techniques, as different approaches have been used to increase the diameter of coagulation. Moreover, the relatively short follow-up time reported for some of the systems does not facilitate helpful comparison. However, some preliminary considerations should be stressed. Radio-frequency ablation, like PEI, demonstrated less effectiveness in the treatment of contiguous multinodular HCC, probably because of the fibrosis surrounding the small neoplastic nodules, which may influence heat diffusion and thereby limit treatment effectiveness $(19,22)$. Moreover, by using this method in neoplastic tissue of the most common type (ie, nodular), one can generally reproduce the 


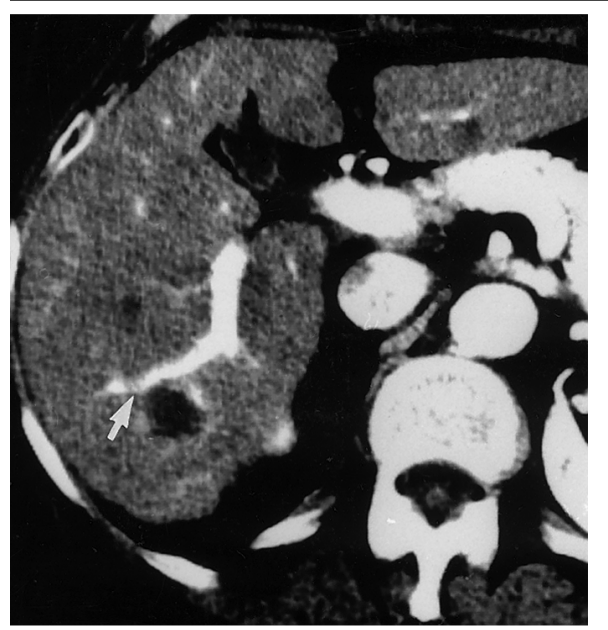

a.

Figure 4. CT scans show residual viable tissue after the procedure. (a) Transverse arterial phase CT scan obtained before LTA shows a hypoattenuating 2.5-cm-diameter nodular HCC (arrow) in segment 6. (b) Transverse arterial phase CT scan obtained 3 months after the first treatment session (energy delivered, 14,400 J) shows an area of residual viable tissue (arrowhead) within the original lesion contiguous to the coagulation. The size and shape of the area of necrosis produced by LTA together with the residual neoplastic tissue are the size and shape of the original neoplasm. (c) Transverse CT scan obtained after the second treatment session (energy delivered, 14,400 J) shows complete coagulation of the residual viable tissue with an area of necrosis (arrowheads) larger than the original lesion. size and shape of the necrotic area so that it is similar to that of the tumor, as if a mold of the tumor had been made (oven effect) (22). The same authors (38) confirmed these results in another study, explaining that with HCC it is generally sufficient to treat just the tumor itself, and that the oven effect may protect surrounding structures.

More recently, Rossi et al (39), by using the radio-frequency interstitial tumor ablation, or RITA, with a four-pronged umbrella needle system (RITA Medical Systems, Mountain View, Calif) after occlusion of the tumor blood supply, confirmed again that lethal temperatures are reached just inside the HCC nodule, whereas nonlethal temperatures are detected in the hepatic tissue immediately surrounding the nodule, and therefore, the resultant necrosis selectively involves only the HCC nodule and reproduces the nodule shape. The same authors (39) reported that in their study, the local recurrences may have been due to the unsatisfactory diffusion of heat within the HCC nodule, as well as extracapsular invasion or daughter nodules outside the original nodule. These results seem to indicate that radiofrequency ablation is unable to induce a volume of necrosis larger than the original lesion and thus obtain the complete destruction of neighboring neoplastic or preneoplastic nests. However, a longer follow-up is needed for a better understanding of the outcomes of these techniques, and more valid data are needed to compare the different options.

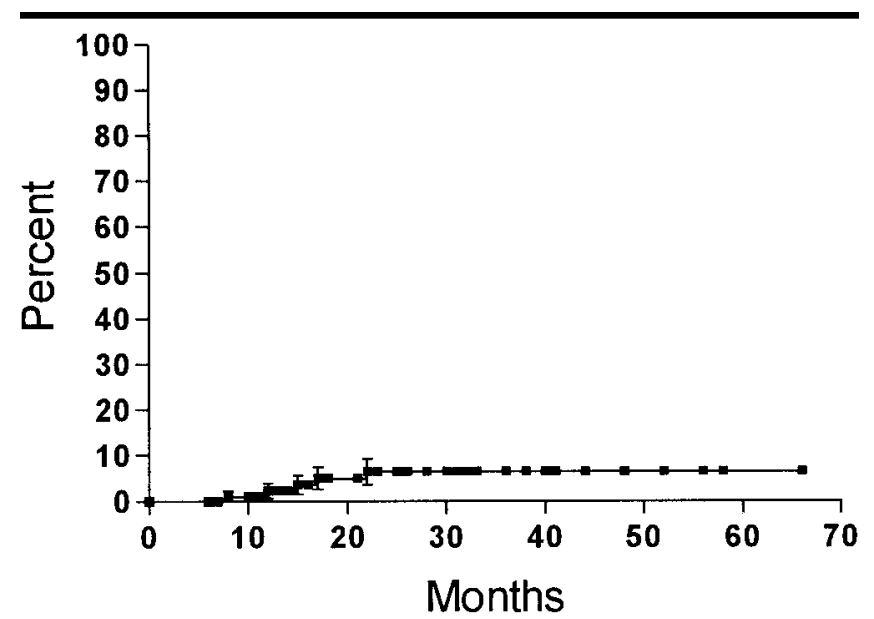

Figure 5. Graph shows 5-year local recurrence rates, calculated with the Kaplan-Meier method, for 74 patients with small HCCs treated with LTA. All recurrences were observed within 2 years.

Recurrence of new HCCs in locations far from the original tumor was frequent during follow-up; this was probably due to multiple metachronous or unnoticed synchronous lesions or to intrahepatic metastases that were not detected at imaging (37). Such new lesions are part of the natural history of HCC in cirrhosis. The entire cirrhotic liver contains regenerative nodules, and hepatocarcinogenesis is probably a stepwise process from a regenerative nodule to an adenomatous hyperplasia and then to HCC (40). Thus, the entire liver is ready for the development of multiple HCCs, and the first lesion to be detected is only the beginning of a multi- focal disease with several tumor clones $(40,41)$.

The survival rates in our study were comparable to those of other studies $(12,17)$. However, we stress that some reports involved patients who received diagnosis and were staged a few years ago when imaging techniques were less accurate. In our view, the use of these less accurate imaging techniques to verify local control of the disease may have caused an underestimation of the rates of local recurrence and of the short-, mid-, and long-term results with PEI. Hence, this limitation must also be taken into account when our results are compared with those of other authors. 


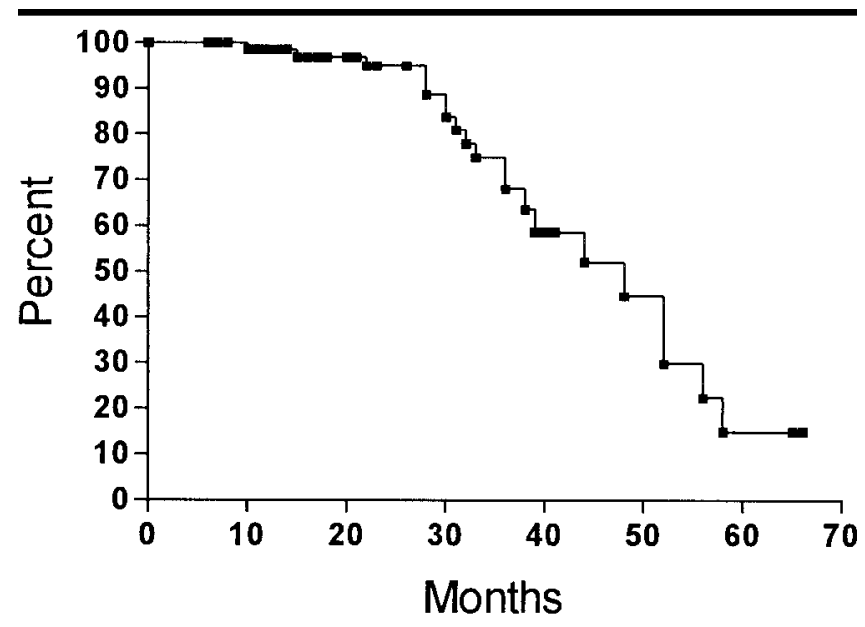

Figure 6. Graph shows 5-year cumulative survival curves, calculated with the Kaplan-Meier method, for 74 patients with small HCCs treated with LTA. The survival rates were $99 \%, 68 \%$, and $15 \%$ at 1,3 , and 5 years, respectively.

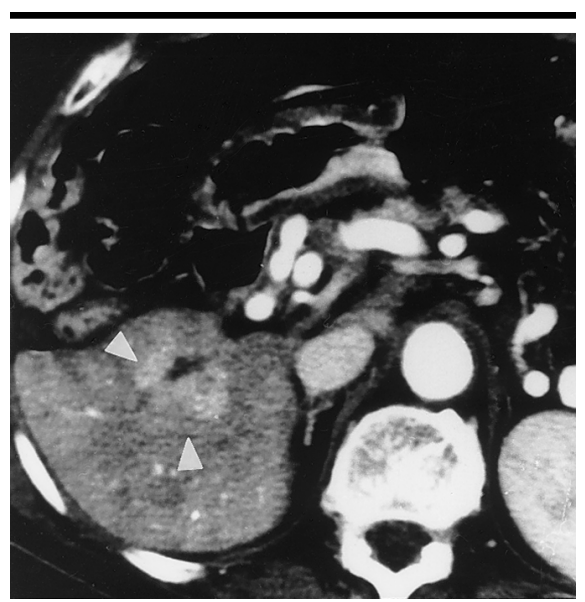

Figure 8. Transverse arterial phase CT scan obtained 17 months after LTA shows hyperattenuating viable tissue (arrowheads) around and within the lesion due to local recurrence.

Thus, there is a need for prospective, randomized, controlled trials with a greater number of patients with predefined characteristics to facilitate the analysis and comparison of the different treatment options offered to patients with HCC.

The best way to ascertain the effectiveness of LTA is to assess the presence of vascular enhancement after contrast material administration at $\mathrm{CT}$, considering that nonenhancement represents absence of viable tissue and thus successful necrosis of the tumor (20-22,25-27). The use of triple-phase helical CT is, in our opinion, essential for assessing treatment effectiveness and completeness and for early detection of tumor recurrence (42). The relationship between the enhancing peripheral halo and LTA treatment de-

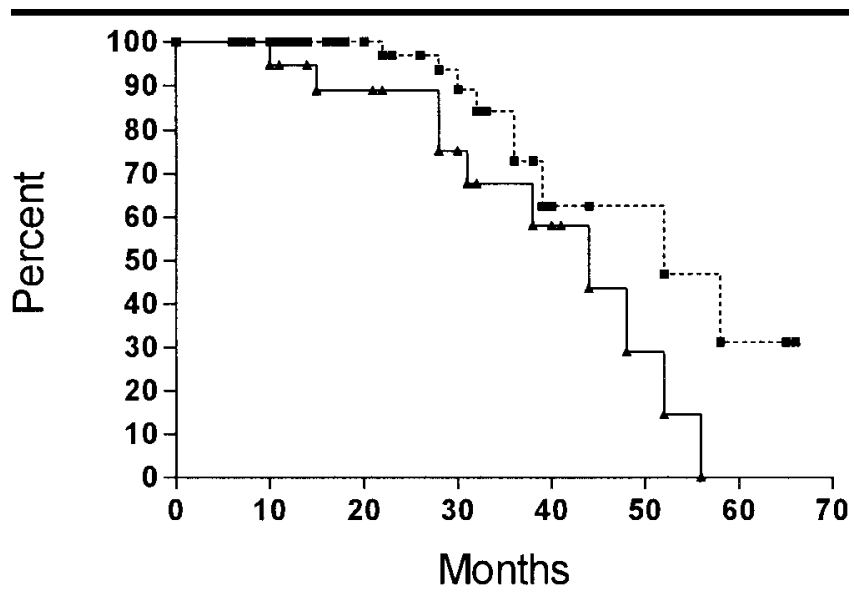

Figure 7. Graph shows 5-year cumulative survival curves, calculated with the Kaplan-Meier method, in accordance with the degree of liver dysfunction on the basis of Child-Pugh classification. The difference was not statistically significant $(P=.052)$. The survival rates were $100 \%, 73 \%$, and $31 \%$ in patients with Child-Pugh class A (ם) and $95 \%, 68 \%$, and $0 \%$ in patients with Child-Pugh class B $(\mathbf{\Delta})$ at 1,3 , and 5 years, respectively. serves some comment, and in our view, the attenuation behavior, with its segmental appearance after bolus injection, can be explained. The hyperattenuating halo around the coagulated tissue that is thought to be related to hyperemia is probably due to a rapid change in hepatic arterial flow in response to thermal injury. Thermal ablation induces a marked increase in arterial perfusion in the surrounding liver within a few minutes after the start of treatment. The heated area of necrosis can cause hepatic segmental venous occlusion, and in this condition, the portal veins become draining veins and the occluded region is supplied with arterial blood alone (43). This phenomenon causes hyperattenuation in the occluded area at CT since arterial flow itself increases and the contrast medium is less diluted there. CT acquisition with rapid sequential sections makes it possible to evaluate these changes.

Determination of the volume of the area to be heated is complex and depends on the laser power, the laser irradiation time and wavelength of light involved, how the energy reaches the tissue, and such tissue factors as optical and thermal properties $(22-27,29,44)$. Since different types of tissue have different optical characteristics, the choice of a specific laser treatment must be related to the tissue type involved (44). Because of these factors, according to a few authors (27), it is not practical to establish a fixed deposition of laser energy for any given tumor size; LTA of tumors of the same size may result in variable necrosis, despite the use of the same amount of laser energy. On the basis of our experience, however, we believe that the therapeutic parameters are $5 \mathrm{~W}$ of laser power administered so as to reach simultaneously a total energy of 7,200 J to an ellipsoid volume ranging from 8 to $18 \mathrm{~mL} \pm 3.9$ (mean, $13.4 \mathrm{~mL}$ ) for a single tumor treatment and ranging from 12 to $40 \mathrm{~mL} \pm 11.5$ (mean, $21.2 \mathrm{~mL}$ ) for two tumor treatments with a total energy deposition of $14,400 \mathrm{~J} \mathrm{(32)}$ and a procedure time of 6 and 12 minutes, respectively.

Although no formal cost-effectiveness analysis was performed as part of this work, it should be pointed out that for LTA, the laser generator costs were $\$ 40,000-\$ 45,000$ and the costs for a set of fibers were only $\$ 1,000-\$ 1,500$, but this set can be used to treat up to 50 patients (45).

In conclusion, LTA is a safe and effective percutaneous technique for the treatment of primary liver tumors. However, further research in this field is needed to elucidate (a) the exact correlation between histopathologic structure and laser energy and (b) the correlation between hepatic blood flow and the actual extent of the area of necrosis (46).

Acknowledgment: We thank Manuela Latini and Laura Morelli for their help in the care of patients during conscious sedation.

\section{References}

1. Oka H, Kuroda N, Kim K, et al. Prospective study of early detection of hepatocel- 
lular carcinoma in patients with cirrhosis. Hepatology 1990; 12:680-687.

2. Zoli M, Magalotti D, Bianchi G, et al. Efficacy of a surveillance program for early detection of hepatocellular carcinoma. Cancer 1996; 78:977-985.

3. Makuuchi M, Kosuge T, Takayama T, et al. Surgery for small liver cancers. Semin Surg Oncol 1993; 9:298-304.

4. Bruix J, Cirera I, Clavet X, et al. Surgical resection and survival in western patients with hepatocellular carcinoma. J Hepatol 1992; 15:350-355.

5. Castells A, Bruix J, Bru C, et al. Treatment of small hepatocellular carcinoma in cirrhotic patients: a cohort study comparing surgical resection and percutaneous ethanol injection. Hepatology 1993; 18:1121-1126.

6. Rolles K. Transplantation of liver cancer. In: Okuda K, Tabor E, eds. Liver cancer. New York, NY: Churchill Livingstone, 1997; 531-535.

7. Matsui O, Kadoya M, Yoshikawa J, et al. Small hepatocellular carcinoma: treatment with subsegmental transcatheter arterial embolization. Radiology 1993; 188:79-83.

8. Bronowicki JP, Vetter D, Doffoel M. Chemoembolization of hepatocellular carcinoma. In: Okuda K, Tabor E, eds. Liver cancer. New York, NY: Churchill Livingstone, 1997; 463-469.

9. Silverman SG, Tuncali K, Adams DF, et al. MR imaging-guided percutaneous cryotherapy of liver tumors: initial experience. Radiology 2000; 217:657-664.

10. Honda N, Guo Q, Uchida H, et al. Percutaneous hot saline injection therapy for hepatic tumors: an alternative to percutaneous ethanol injection therapy. Radiology $1994 ; 190: 53-57$.

11. Ohnishi $\mathrm{K}$, Yoshioka H, Ito $\mathrm{S}$, et al. Prospective randomized controlled trial comparing percutaneous acetic acid injection and percutaneous ethanol injection for small hepatocellular carcinoma. Hepatology 1998; 27:67-72.

12. Livraghi T, Giorgio A, Marin G, et al. Hepatocellular carcinoma and cirrhosis in 746 patients: long-term results of percutaneous ethanol injection. Radiology 1995; 197:101-108.

13. Higuchi T, Kikuchi M, Okazaki M. Hepatocellular carcinoma after transcatheter hepatic arterial embolization: a histopathologic study of 84 resected cases. Cancer 1994; 73:2259-2267.

14. Shiina $\mathrm{S}$, Tagawa $\mathrm{K}$, Unuma $\mathrm{T}$, et al. Percutaneous ethanol injection therapy for hepatocellular carcinoma: a histopathologic study. Cancer 1991; 68:1524-1530.

15. Ishii $H$, Okada $S$, Nose $H$, et al. Local recurrence of hepatocellular carcinoma after percutaneous ethanol injection. Cancer 1996; 77:1792-1796.

16. Ebara M, Ohto M, Sugiura N, et al. Percutaneous ethanol injection for the treatment of small hepatocellular carcinoma: study of 95 patients. J Gastroenterol Hepatol 1990; 5:616-626.
17. Shiina S, Tagawa K, Niwa Y, et al. Percutaneous ethanol injection therapy for the treatment of hepatocellular carcinoma: results in 146 patients. AJR Am J Roentgenol 1993; 160:1023-1028.

18. Ishii $\mathrm{H}$, Okada $\mathrm{S}$, Okusaka $\mathrm{T}$, et al. Needle tract implantation of hepatocellular carcinoma after percutaneous ethanol injection. Cancer 1998; 82:1638-1642.

19. Yamashita Y, Matsukawa T, Arakawa A, et al. US-guided liver biopsy: predicting the effect of interventional treatment of hepatocellular carcinoma. Radiology 1995; 196:799-804.

20. Rossi S, Di Stasi M, Buscarini E, et al. Percutaneous RF interstitial thermal ablation in the treatment of hepatic cancer. AJR Am J Roentgenol 1996; 167:759-768.

21. Solbiati L, Goldberg SN, Ierace T, et al Hepatic metastases: percutaneous radiofrequency ablation with cooled-tip electrodes. Radiology 1997; 205: 367-373.

22. Livraghi T, Goldberg SN, Lazzaroni S, et al. Small hepatocellular carcinoma: treatment with radio-frequency ablation versus ethanol injection. Radiology 1999; 210:655-661.

23. Murakami R, Yoshimatsu S, Yamashita Y, et al. Treatment of hepatocellular carcinoma: value of percutaneous microwave coagulation. AJR Am J Roentgenol 1995; 164:1159-1164.

24. Matthewson K, Coleridge-Smith P, O'Sullivan $\mathrm{P}$, et al. Biological effects of intrahepatic neodymium:yttrium-aluminium-garnet laser photocoagulation in rats. Gastroenterology $1987 ; 93: 550-557$.

25. Huang GT, Wang TH, Sheu JC, et al. Lowpower laserthermia for the treatment of small hepatocellular carcinoma. Eur J Cancer 1991; 27:1622-1627.

26. Dowlatshahi K, Bhattacharya AK, Silver $\mathrm{B}$, et al. Percutaneous interstitial laser therapy of a patient with recurrent hepatoma in a transplanted liver. Surgery 1992; 112:603-606.

27. Amin Z, Donald JJ, Masters A, et al. Hepatic metastases: interstitial laser photocoagulation with real-time US monitoring and dynamic CT evaluation of treatment. Radiology 1993; 187:339-347.

28. Vogl TJ, Muller PK, Hammerstingl R, et al. Malignant liver tumors treated with MR imaging-guided laser-induced thermotherapy: technique and prospective results. Radiology 1995; 196:257-265.

29. Pacella CM, Bizzarri G, Ferrari FS, et al. Interstitial photocoagulation with laser in the treatment of liver metastases. Radiol Med 1996; 92:438-447.

30. Kraft M, Arellano RS, Mueller PR. Conscious sedation for the non-anesthesiologist: a primer. Semin Intervent Radiol 1999; 16:89-99.

31. Steger AC, Lees WR, Shorvon P, Walmsley $\mathrm{K}$, Bown SG. Multiple-fibre low-power interstitial laser hyperthermia: studies in the normal liver. Br J Surg 1992; 79:139-145.

32. Pacella CM, Rossi Z, Bizzarri G, et al. Ul- trasound-guided percutaneous laser ablation of liver tissue in a rabbit model. Eur Radiol 1993; 3:26-32.

33. Heisterkamp J, van Hillegersberg R, Sinofsky EL, et al. Heat resistant cylindrical diffuser for interstitial laser coagulation: comparison with a bare-tip fibre in ex vivo porcine liver. Lasers Surg Med 1997; 20:304-309.

34. Miller AB, Hoogstraten B, Staquet M, et al. Reporting results of cancer treatment. Cancer 1981; 47:207-214.

35. Heisterkamp J, van Hillegersberg R, Ijzermans JNM. Interstitial laser coagulation for hepatic tumours. Br J Surg 1999; 86:293-304.

36. Vilana R, Bruix J, Bru C, et al. Tumor size determines the efficacy of percutaneous injection for the treatment of small hepatocellular carcinoma. Hepatology 1992; 16:353-357.

37. Utsunomiya T, Matsumata T, Adachi E, et al. Limitations of current preoperative liver imaging techniques for intrahepatic metastatic nodules of hepatocellular carcinoma. Hepatology 1992; 16:694-701.

38. Livraghi T, Goldberg SN, Lazzaroni S, et al. Hepatocellular carcinoma: radio-frequency ablation of medium and large lesions. Radiology 2000; 214:761-768.

39. Rossi S, Garbagnati F, Lencioni R, et al. Percutaneous radio-frequency thermal ablation of nonresectable hepatocellular carcinoma after occlusion of tumor blood supply. Radiology 2000; 217:119-126.

40. Takayama T, Makuuchi M, Hirohashi S, et al. Malignant transformation of adenomatous hyperplasia to hepatocellular carcinoma. Lancet 1990; 336:1150-1153.

41. Takenaka K, Adachi E, Nishizaki T, et al. Possible multicentric occurrence of hepatocellular carcinoma: a clinicopathological study. Hepatology 1994; 19:889-894.

42. Pacella CM, Anelli V, Bizzarri G, et al. Evaluation of the vascular pattern of hepatocellular carcinoma with dynamic computed tomography and its use in identifying optimal temporal windows for helical computed tomography. Eur Radiol 1998; 8:30-35.

43. Murata S, Itai Y, Asato M, et al. Effect of temporary occlusion of hepatic vein on dual blood supply in the liver: evaluation with spiral CT. Radiology 1995; 197:351-356.

44. van Hillegersberg $\mathrm{R}$, Pickering JW, Aalders $\mathrm{M}$, et al. Optical properties of rat liver and tumor at $633 \mathrm{~nm}$ and $1064 \mathrm{~nm}$ : photofrin enhances scattering. Lasers Surg Med 1993; 13:31-39.

45. Dodd JD, Soulen MC, Kane RA, et al. Minimally invasive treatment of malignant hepatic tumors: at the threshold of a major breakthrough. RadioGraphics 2000; 20:9-27.

46. Heisterkamp J, van Hillegersberg R, Mulder PGH, et al. Importance of eliminating portal flow to produce large intrahepatic lesions with interstitial laser coagulation. Br J Surg 1997; 84:1245-1248. 